\title{
HYPOTHESIS
}

\section{Is rheumatoid arthritis a failure of B cell death in synovium?}

\author{
Jo C W Edwards, Jo Cambridge
}

Why autoantibodies and joints?

The dominant features of rheumatoid arthritis (RA) are synovitis of joints, tendon sheaths, and bursae, and the production of a wide range of autoantibodies, including rheumatoid factor. ${ }^{1}$ Any hypothesis for the pathogenesis of RA must marry these two, apparently unrelated, aspects. This has proved difficult, but recent studies suggest a model which may provide a solution.

The only spontaneous animal model approximating to human RA is the MRL/pr mouse, which has a defect in programmed cell death. ${ }^{2}$ The MRL/pr mouse phenotype differs from RA not only in having additional features of systemic lupus, but in having massive lymphadenopathy, with a dominant accumulation of $T$ cells; in RA, abnormal numbers of lymphocytes are seen chiefly in synovium. Moreover, though the synovial $\mathrm{T}$ cell infiltrate is not very different from that seen in mechanically induced synovitis (osteoarthritis), the presence of $B$ cell germinal centres in a proportion of cases is almost unique to RA. ${ }^{3}$

It is proposed that RA is a failure of cell death, but one which is lineage specific to $B$ cells and site specific to synovium. The basis for this lineage and site specificity lies in a fortuitous similarity between follicular dendritic reticulum cells (FDRC) and synovial intimal cells.

Normal B lymphocytes are believed to die unless they receive certain signals while in contact with FDRC. If synovial intimal cells could act as surrogate FDRC, then B lymphocytes that would normally die might survive in a non-lymphoid environment, beyond the control of a full complement of regulatory $T$ cells. This could lead to the production of a range of inappropriate antibodies, including autoantibodies. It is suggested that two molecules, vascular cell adhesion molecule-1 (VCAM-1) and complement decay accelerating factor (DAF, CD55), may play a central role in such a process.

\section{B lymphocyte survival}

Division of

Rheumatology, University College London,

Arthur Stanley House, London W1P 9PG, United Kingdom J C W Edwards G Cambridge Accepted for publication 8 June 1995
B cell survival and proliferation is normally confined to the germinal centres of lymphoid tissue. ${ }^{4}$ Four associated cell types are believed to have a controlling influence: FDRC carry antigen on their surface and present it to B cells; CD4 positive $T$ helper cells assist the expansion of specific $B$ cell clones; interdigitating dendritic cells (IDC) present antigen to the $T$ cells surrounding the germinal centre; germinal centre macrophages dispose of B cells undergoing programmed cell death. ${ }^{4}$

Macrophages, IDC like cells, and $T$ cells migrate through most normal non-lymphoid tissues, including synovium, where macrophages are numerous, and IDC like cells and $T$ cells occur in small numbers. ${ }^{5-7}$ FDRC are not present in normal non-lymphoid tissue.

The dendrites of FDRC make intimate contact with and interact with all germinal centre lymphocytes, through the expression of VCAM-1. B cell proliferation is dependent on VCAM- 1 binding to its ligand, $\alpha \mathbf{4} \beta 1$ integrin, on $B$ cells and probably also on adjacent CD4 T cells. ${ }^{89}$

FDRC encourage $B$ cell proliferation by presenting antigen in the form of immune complexes bound to immunoglobulin $\mathrm{Fc}$ receptors $(\mathrm{FcR})$, in particular the low affinity FcRII, rather than class II. ${ }^{4}$ They are, in this and other respects, unrelated to other 'dendritic' cells.

The presence of complement appears to be essential to FDRC-B cell interaction. However, in the presence of immune complexes, complement could pose a threat to the cells of the germinal centre. The ability of complement to kill nucleated cells in vivo is a matter of debate. Nevertheless, there is evidence that control mechanisms exist which protect cells by inhibiting the formation of complement membrane attack complex. Thus the implication is that complement might be able to kill FDRC, B cells, or both, in the absence of these control mechanisms.

Within germinal centres, complement activity appears to be controlled partly by the presence of complement receptors (CR1, CR2) on lymphocytes and FDRC, but also by high levels of expression on FDRC of DAF which accelerates complement decay. ${ }^{10}$ In vitro studies suggest that DAF is critical to protection of lymphocytes against complement attack, and may also protect against natural killer cell killing. ${ }^{11}$

B cell survival is also dependent on the presence on B cells of CD40, and on T cells of its ligand, CD40L. ${ }^{4}$ Recent discussion of $B$ cell survival has focused on this interaction, but as $\mathrm{T}$ cells occur in most tissues, the lack of CD40-CD40L interactions in non-lymphoid tissues may relate as much to the absence of VCAM-1 positive cells capable of bringing $B$ and $T$ cells together and allowing them to interact, as to a lack of CD40 or CD40L.

In simple terms, B cell survival appears to be limited to germinal centres by the need for 
nearby cells carrying antigen presented on FcRII, VCAM-1 to hold the B cells together with CD4 $\mathrm{T}$ cells, and both complement receptors and high levels of DAF to control complement activation. (It may be worth noting that in gut associated lymphoid tissue, VCAM-1 positive DAF positive FDRC appear not to operate. ${ }^{812}$ )

\section{Cross talk between adhesion systems}

An intriguing feature of cell surface molecules involved in adhesion and signalling is that their usage appears to have evolved, as predicted by Darwinism, on a truly 'free market' basis. Individual adhesion molecules are used for multiple unrelated functions in developing and adult tissues. Thus VCAM-1 is involved in fusion of myoblasts, $B$ cell proliferation, leucocyte-endothelial interactions and an unknown function in synovial intima. ${ }^{13} 14$ This multiple usage raises the possibility of cross talk between unrelated functions in unrelated tissues.

\section{Synovial intimal cells}

Synovial intimal fibroblasts (SIF) are unusual amongst fibroblasts in that they express constitutively, and at high level, a number of molecules which are normally associated with other cell types or states of activation. ${ }^{15}$ These include VCAM-1 and DAF. Moreover, the amounts of VCAM-1 are much greater than those seen on blood vessels and those of DAF are much greater than those seen on vessels or leucocytes. ${ }^{14} 1617$ It would not be unreasonable to think that, at high dilution, both anti-DAF and anti-VCAM-1 antibodies were virtually specific to SIF and FDRC (and Bowman's capsule!) on tissue sections. To our knowlege, no other fibroblast like cells show similar coexpression to these molecules.

SIF do not express FcRII or complement receptors. However, synovial intimal macrophages (SIM) do, and in abundance. ${ }^{18} 19$ Indeed, in inflamed synovium at least, SIM express relatively more $\mathrm{FcRII}$ than $\mathrm{FcRI}$ when compared with other macrophage subpopulations. ${ }^{20}$ Complement can bind to SIM complement receptors, and large amounts of complement have been seen to be concentrated in the intima in osteoarthritic, in addition to rheumatoid, synovium, in association with DAF. ${ }^{16}$ Thus the synovial intima may provide an environment remarkably similar to that encountered by $B$ and $T$ lymphocytes in germinal centres (figure).

\section{Could SIF or SIM act as FDRC?}

The fact that molecules expressed on germinal centre FDRC are shared by two cell types in synovial intima may limit any functional similarity between the two environments. However, there are some further curious similarities between FDRC and intimal cells. Both have been the subject of longstanding debate about bone marrow or tissue fixed origin. FDRC are currently believed to be of local fibroblast origin, ${ }^{21}$ while current opinion favours a local origin for SIF, and a bone marrow origin for SIM. ${ }^{22}$ However, many groups have described intermediate cells. Our own view is that evidence for intermediate forms is weak. Nevertheless, at least in inflamed tissue, fusion polykaryons (giant cells) are common in synovial intima. ${ }^{23}$ Most appear to be either SIF or SIM homopolykaryons, but a few could be interpreted as having the mixed features expected of heteropolykaryons (hybrids). FDRC often have two or three nuclei: ${ }^{21}$ three nuclei suggests fusion. It is conceivable that arguments about the origins and surface molecule status of FDRC indicate that they too are, at least sometimes, heteropolykaryons.

\section{Why do we not all have RA?}

If synovial intimal cells were truly functionally equivalent to FDRC, then B cells, in the presence of attendant $T$ cells, could survive and proliferate in joints and we should all have RA. The predominance of $T$ cells in RA synovium ${ }^{24}$ would be predicted, as proliferating B cells, whether normal or of a well differentiated malignant phenotype, gather around them much larger numbers of $T$ cells. That most of us do not get RA suggests either that the appropriate mixture of cells does not come into close contact often enough in normal synovium, or that the synovial intimal environment normally falls short of the germinal centre requirements in some other way. Normal synovium contains a very few $T$ cells and scarcely any B cells, ${ }^{7}$ but in osteoarthritic joints large numbers of lymphocytes can be present. It seems more likely that one or more other cofactors are missing from synovial intima, and that RA will only develop if these cofactors are induced. That they can be induced is demonstrated by the presence of fully formed VCAM-1 positive DAF positive FDRC within germinal centres in a proportion of rheumatoid synovia. ${ }^{14}$ An obvious candidate cofactor is the surface molecule DRC1, the function of which is not yet known and which is present on normal and ectopic FDRC, ${ }^{14}{ }^{25}$ but not on normal synovial intima. However, it is of note that B lymphocyte clusters form around VCAM-1 positive cells in rheumatoid synovium, even in the absence of DRC1 expression. $^{14}$

SIF may be inhibited from acting as FDRC by associated hyaluronan, ${ }^{26}$ possibly bound to the cell via CD44. Both molecules include charged glycosaminoglycan residues, which may inhibit lymphocyte-accessory cell interactions. $^{27}$ However, a proportion of SIF express VCAM-1 while failing to show evidence of hyaluronan secreting capacity, ${ }^{14}$ and perhaps these cells are more likely to interact with B cells.

\section{Induction of FDRC associated genes in synovial cells}

Ectopic FDRC in rheumatoid synovia lie within deep tissue, which normally contains no 

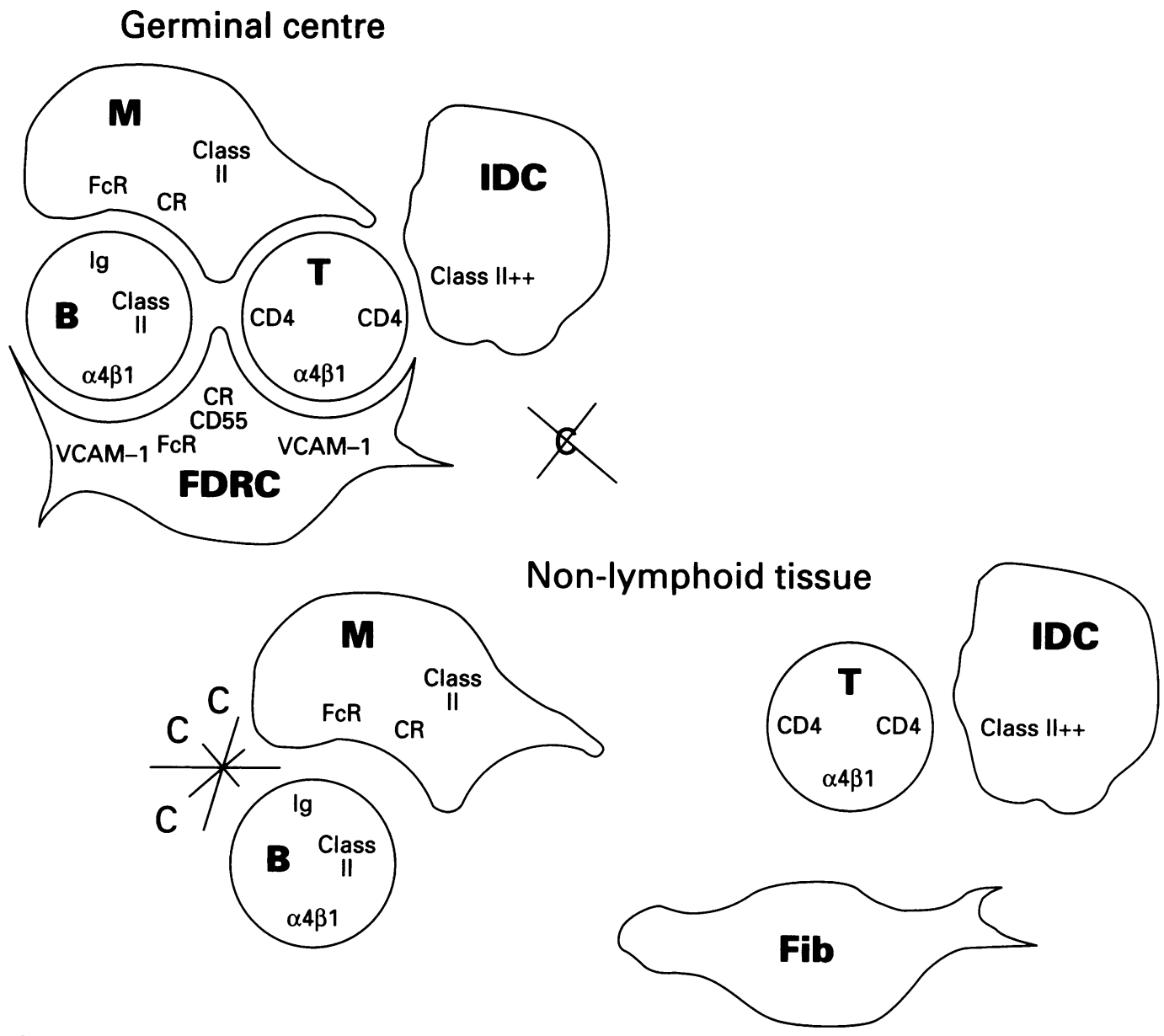

Synovial intima

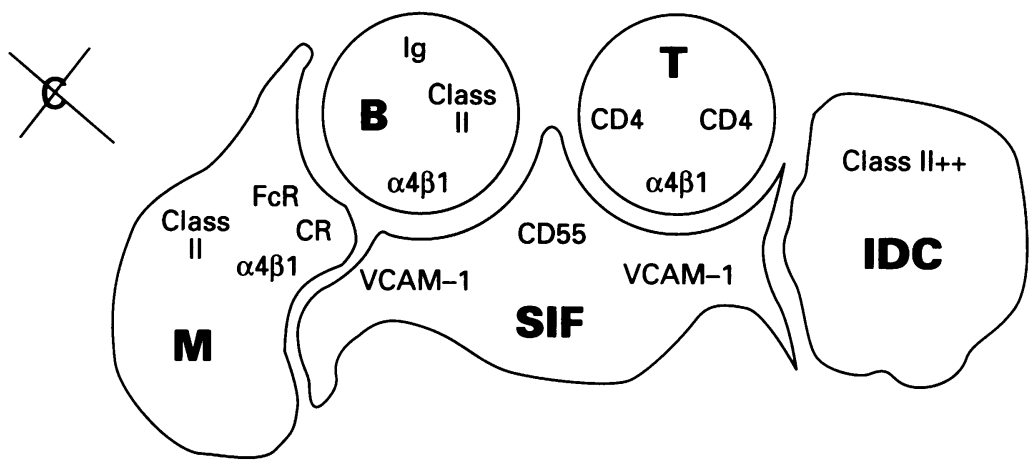

The cellular environments associated with germinal centres, non-lymphoid tissue, and the synovial intima. $\alpha 4 \beta 1=\alpha 4 \beta 1$ integrin; $B=B$ cell; $C=$ complement activation; $C R=$ complement receptor; $F c R=$ immunoglobulin $F c$ receptor; Fib = fibroblast $; I D C=$ interdigitating dendritic cell; $M=$ macrophage; $S I F=$ synovial intimal fibroblast $;=T$ cell.

VCAM-1 or DAF cells, distant from SIF. This raises the question of the relationship between deep and intimal synovial fibroblasts in terms of their origins and ability to express specific gene products. SIF interacting with $B$ cells could be engulfed into the stroma as disease develops, but deep cells could also take on SIF features under the appropriate conditions.

In early embryonic development, cells of the interzone (which is destined to become both deep and intimal synovium) strongly express CD44. ${ }^{28}$ As the interface between interzone and cartilage becomes clear, cells at the interface begin to express DAF (manuscript submitted for publication). The same cells then show an increase in hyaluronan production and the joint cavity is formed. This leaves an intimal layer of DAF cells on the surface of the synovium, which then becomes distinct from deeper interzone in further respects. ${ }^{28}$ CD44 and $\beta 1$ integrin expression decrease in the deeper layers, but increase on the intima. Much later, perhaps postnatally, SIF become VCAM-1 positive.

This sequence of events suggests a complex cascade of transcriptional control linking the expression of these molecules. In vitro CD44 expression is maintained on synovial fibroblasts, ${ }^{29}$ but DAF and VCAM-1 are lost, becoming dissociated in individual cells in the process. However, synovial fibroblasts will reexpress VCAM-1 more readily than dermal fibroblasts in response to tumour necrosis factor $\alpha{ }^{30}{ }^{31}$ Current evidence suggests that if implants are inserted into connective tissue, they become surrounded by a membrane of tissue which will carry DAF and VCAM-1 positive cells if the implant is placed at a 
pre-existing synovial site, but not if placed elsewhere. $^{22} 3233$ Thus it appears that synovial fibroblasts in general may express certain gene products under conditions which would not lead to expression in other tissues.

The occurrence of FDRC in association with metastatic $B$ follicular lymphoma cells suggests that FDRC will differentiate from local fibroblasts in any tissue if a strong enough signal is received from lymphoid or other cells. The suggestion is made that, within synovium, this differentiation is facilitated for all fibroblasts, thus making the tissue a locus minores resistentiae for extralymphoid B cell survival. This would explain the old immunologists' adage that the best way to raise an antibody is to inject antigen into a joint.

\section{A disaster waiting to happen}

It is proposed that rheumatoid arthritis is a 'disaster waiting to happen' thus. After a trivial initial stimulus, a B lymphocyte clone finds conditions in synovium which allow it to survive and proliferate. Antibodies of various specificities may be formed after somatic mutation without adequate $\mathrm{T}$ cell control. The process may abort, or progress, via the dissemination of autoantibody based complexes. These may encourage $B$ cell influx into other joints and may be the basis of complications such as vasculitis. Once formed, FDRC are very long lived, with the ability to carry long term immunological memory in the form of surface bound antigen, ${ }^{4}$ providing a basis for chronicity. The process may only regress if $\mathrm{T}$ cells accumulate in large enough numbers in synovium to re-establish control. There is no doubt that large numbers of $T$ cells do accumulate in rheumatoid joints and at least one observer has linked their numbers to remission of tissue destruction. ${ }^{34}$

Rheumatoid factor may contribute to immune complex mediated events, but the model does not require that it should always be present (and it is not). The association of RA with major histocompatibility complex (MHC) class II allotype ${ }^{35}$ is consistent with the involvement of class II in interactions with 'helper' CD4 $\mathrm{T}$ cells. There are also complex associations, in terms of both gene mapping and coexpression of gene products, between MHC and complement related genes, suggesting that the class II association may have a more subtle basis. $^{3637}$ A wide range of defects could overcome the threshold for B cells survival in synovium, and it may be that the unaccounted for genetic component of the condition relates to multiple variations in regulation of gene expression. The overlap between RA and systemic lupus also conjures up tantalising analogies between possible complement regulatory defects in the two conditions.

Many other clinical and laboratory data would appear to be consistent with the model. Rheumatoid nodules remain a puzzle, but a number of possible explanations for them can be proposed. The validity of the model can be tested by manipulation of fibroblast gene expression in vitro to assess the true require- ments for FDRC formation and interaction of fibroblast derived cells with immature $B$ lymphocytes. Therapy could be directed at ligands involved in B cell-FDRC interactions or at regulation of DAF. Certainly, there are new avenues to explore.

The author would like to acknowledge the support of the Arthritis and Rheumatism Council. The proposed hypothesis was made possible by the hard work of Ms Linda Wilkinson, Dr Andy Pitsillides, and Dr Jennifer Worrall, in particular.

1 Klippel J H, Dieppe P A (eds). Rheumatology. London: Mosby-Year Book, 1994; 3.3.1-9.14

2 Watanabe-Fukunaga R, Brannan C I, Copeland N G, Jenkins N A, Nagata S. Lymphoproliferation disorder in mice explained by defects in Fas antigen that mediates apoptosis. Nature 1992; 356: 314-7.

3 Gardner D L. Rheumatoid arthritis: cell and tissue pathology. In: Gardner D L, ed. Pathological basis of connective tissue diseases. London: Edward Arnold, 1992; 444-526.

4 McLennan I C. Germinal centres. Annu Rev Immunol 1994; 12: $117-39$.

5 Edwards J C W. Structure of the synovial lining. In: Henderson B, Edwards J C W, eds. The synovial lining in health and disease. London: Chapman and Hall, 1987; $29-40$.

6 Wilkinson L S, Worrall J G, Sinclair H S, Edwards J C W. Immunohistochemical reassessment of accessory cell populations in normal and diseased human synovium. $\mathrm{Br}$ f Rheumatol 1990; 29: 259-63.

7 Lindblad S, Hedfors E. The synovial membrane of healthy individuals-immunohistochemical overlap with synovitis. Clin Exp Immunol 1987; 69: 41-7.

8 Freedman A S, Munro J M, Rice G E, et al. Adhesion of human $B$ cells to germinal centres in vitro involves VLA-4 human B cells to germinal centres in vitro involve

9 Rice G E, Munro J M, Corless C, et al. Vascular and nonvascular expression of INCAM-110 a target for mononuclear leucocyte adhesion in normal and inflamed nuclear leucocyte adhesion in normal and

10 Lampert I A, Schofield J B, Amlot P, Van Noorden S. Protection of germinal centres from complement attack. f Pathol 1993; 170: 115-20.

11 Nicholson Weller A, Wang C E. Structure and function of decay accelerating factor CD 55. $\mathcal{F}$ Lab Clin Med 1994; 23 : 485-91.

12 Medof M E, Walter E I, Rutgers J L, Knowles D M, Nussenzweig V. Identification of the complement decay accelerating factor on epithelium and glandular cells and in body fluids. F Exp Med 1987; 165: 848-64.

13 Iademarco M F, McQuillan J J, Dean D C. Vascular cell adhesion molecule 1 : contrasting transcriptional mechanisms in muscle and endothelium. Proc Natl Acad Sci 1993; 90: 3943-7

14 Wilkinson L S, Edwards J C W, Poston R, Haskard D O. Cell populations expressing VCAM-1 in normal and diseased synovium. Lab Invest 1993; 68: 82-8.

15 Edwards J C W. Fibroblast-like synovial lining cells. In Henderson B, Edwards J C W, Pettifer E R, eds. Mechanisms and models in rheumatoid arthritis. London Academic Press, 1995; 153-61.

16 Tarkowski A, Trollmo C, Seifert P S, Hansson G K Expression of decay accelerating factor on synovial lining Expression of decay accelerating factor on synovial lining
cells in inflammatory and degenerative arthritides. cells in inflammatory and

17 Guc D, Gulati P, Lemercier C, Lappin D, Birnie G D, Whaley $\mathrm{K}$. Expression of the components and regulatory proteins of the alterative complement pathway and the membrane attack complex in normal and diseased synovium. Rheumatol Int 1993; 13: 139-46.

18 Theofilopoulos A N, Carson D A, Tavassoli M, et al. Evidence for the presence of receptors for $\mathrm{C} 3$ and IgG Fc on human synovial cells. Arthritis Rheum 1980; 23: 1-19.

19 Edwards J C W, Sedgwick A D, Willoughby D A. Membrane properties and esterase activity of synovial lining cells: further evidence for a mononuclear phagocyte subpopulation. Ann Rheum Dis 1982; 41: 282-6.

20 Bröker B, Edwards J C W, Fanger M, Lydyard P. The prevalence and distribution of macrophages bearing prevalence and distribution of macrophages bearing 1990; 19: 123-35.

21 Heinen E, Bosseloir A. Follicular dendritic cells: whose children? Immunol Today 1994; 15: 201-4.

22 Edwards J C W. The nature and origins of synovium. 7 Anat 1994; 184: 493-501.

23 Wilkinson L S, Pitsillides A A, Edwards J C W. Giant cells in arthritic synovium. Ann Rheum Dis 1993; 52: 182-4.

24 Janossy G, Duke O, Poulter L W, Panayi G, Bofill M, Goldstein G. Rheumatoid arthritis: a disease of $\mathrm{T}$ lymphocyte- macrophage immunoregulation. Lancet
1981; ii: 839-42.

25 Naiem M, Gerdes J, Abdulaziz Z, Stein H, Mason D Y. Production of a monoclonal antibody reactive with human dendritic reticulum cells and its use in the immunohistological analysis of lymphoid tissue. $f$ Clin Path 1983; 36: 167-75.

26 Worrall J G, Bayliss M T, Edwards J C W. Distribution of hyaluronan in normal and diseased synovium. f Rheumatol 1991; 18: 1466-72. 
27 King P D, Batchelor A H, Lawlor P, Katz D R. The role of $\mathrm{CD} 44, \mathrm{CD} 45, \mathrm{CD} 45 \mathrm{RO}, \mathrm{CD} 46$ and $\mathrm{CD} 55$ as potential anti-adhesion molecules in the binding of potential anti-adhesion molecules in the binding of 13-acetate-differentiated U-937 cells. Eur $f$ Immunol 19-ace 20: 363-8.

28 Edwards J C W, Wilkinson L S, Jones H M, et al. The formation of human synovial joint cavities. $\mathcal{f}$ Anat 1994 ; 185: $355-67$.

29 Henderson K J, Edwards J C W, Worrall J G. Expression of CD44 in normal and rheumatoid synovia and in cultured synovial fibroblasts. Ann Rheum Dis 1994; 53 729-34

30 Morales Ducret J, Wayner E, Elices $M \mathrm{~J}$, et al. $\alpha_{4} / \beta_{1}$ integrin (VLA-4) ligands in arthritis: Vascular cell adhesion molecule expression in synovium and on fibroblast-like synoviocytes. Arthritis Rheum 1992; 149: 1424-31.

31 Marlor C W, Webb D L, Bombara M P, Greve J M Blue $M$ L. Expression of vascular cell adhesion molecule-1 in fibroblast-like synoviocytes after stimulation with tumor necrosis factor. Am F Pathol 1992; 140: 1055-60.
32 Revell P, Al-Saffar N, Fish S, Osei D. Extracellular matrix of the synovial intimal cell layer. [Proceedings of the Second International Meeting on Synovium]. Ann Rheum
Dis 1995; 54: 404-7. 33 Edwards $\mathrm{C}$. W, Wiks

Edwards J C W, Wilkinson L S, Thornhill T S, Lalor P UDPGD activity and VCAM-1 in synovial like periimplant tissues. [Proceedings of the Second International Meeting on Synovium: abstract]. Ann Rheum Dis 1995; 54: 525-60.

34 Fassbender H G. Rheumatoid arthritis. In: Pathology of rheumatic diseases. Berlin: Springer Verlag, 1975; 109-10.

35 Stastny P. Association of the B cell alloantigen DRw4 with rheumatoid arthritis. $N$ Engl F Med 1978; 298: 869-72.

36 Venneker G T, Asghar S S. CD59; a molecule involved in antigen presentation as well as downregulation of membrane attack complex. Exp Clin Immunogenet 1992; 9: $33-47$.

37 Clinek T, Horejsi V. The nature of noncovalent complexes containing glycosyl-phosphatidylinositol-anchored membrane glycoproteins and protein tyrosine kinases. f Immunol 1992; 149: 2262-70. 